\title{
Fatores associados e prevalência de medicamentos prescritos para idosos institucionalizados do extremo norte do Brasil
}

\author{
Associated factors and prevalence of prescription drugs for institutionalized elderly in the \\ far north of Brazil
}
Factores asociados y prevalencia de medicamentos recetados para ancianos institucionalizados en el extremo norte de Brasil

Thais Renata Muniz ${ }^{1 *}$, Paulo Sergio da Silva², Jackeline da Costa Maciel², Georgia Patrícia da Silva Ferko².

\section{RESUMO}

Objetivo: Avaliar a prevalência e fatores associados à prescrição de medicamentos em idosos institucionalizados. Métodos: Trata-se de um estudo de corte transversal, no qual participaram 27 idosos, de ambos os sexos, que residiam na única instituição de longa permanência para idosos no município de Boa Vista, Roraima há mais de trinta dias no período da coleta de dados. Para caracterizar o perfil farmacoterapêutico dos idosos, foram coletados dados por meio da análise de prontuários e prescrições médicas e posteriormente classificados segundo a WHOCC. Por fim os dados foram processados e tabulados através do software Microsoft Office Exce ${ }^{\circledR} 2016$ e analisados com o auxílio do software estatístico R-project. Resultados: Evidenciou-se que todos os idosos faziam uso de pelo menos um medicamento, com prevalência de idosos polimedicados $(88,9 \%)$ e fármacos que atuam no sistema nervoso $(29,6 \%)$, sendo a dipirona o fármaco com maior frequência de prescrição (59,3\%). Conclusão: Esses resultados reforçam a importância do estabelecimento de uma equipe interdisciplinar com foco na qualidade da assistência prestada, e, consequentemente, despertar o interesse para novas investigações sobre o objeto de estudo, haja vista o aumento da expectativa de vida da população.

Palavras-chave: Idoso, Instituição de longa permanência para idosos, Prescrições de medicamentos.

\begin{abstract}
Objective: To evaluate the prevalence and factors associated with medication prescription in institutionalized elderly. Methods: This is a cross-sectional study, in which 27 elderly people, of both sexes, who lived in the sole long-term care institution for the elderly in the municipality of Boa Vista, Roraima, for more than 30 days in the period of the data collection. To characterize the pharmacotherapeutic profile of the elderly, data were collected through the analysis of medical records and medical prescriptions and subsequently classified according to the WHOCC. Finally, the data were processed and tabulated using Microsoft Office Excel® 2016 software and analized with the help of the statistical software R-project. Results: It was evidenced that all the elderly used at least one medication, with a prevalence of polymedicated elderly (88.9\%) and drugs that act on the nervous system (29.6\%), with dipyrone being the drug most frequently prescripted $(59.3 \%)$. These results reinforce the importance of establishing an interdisciplinary team with a focus on the quality of care provided, and, consequently, arousing interest in new investigations on the object of study, given the increase in life expectancy of the population.
\end{abstract}

Key words: Aged, Homes for the aged, Drug prescriptions.

\section{RESUMEN}

Objetivo: Evaluar la prevalencia y factores asociados a la prescripción de medicamentos en ancianos institucionalizados. Métodos: Se trata de un estudio transversal, en el cual 27 personas mayores, de ambos

1 Distrito Sanitário Indígena Leste de Roraima (DSEI LRR), Boa Vista - RR.

*E-mail: thaysrmuniz@hotmail.com

2 Universidade Federal de Roraima (UFRR), Boa Vista - RR.

SUBMETIDO EM: $3 / 2021$ 
sexos, que vivían en la única institución de cuidados de largo plazo para ancianos en el municipio de Boa Vista, Roraima, más de 30 días en el período de recolección de datos. Para caracterizar el perfil farmacoterapéutico del anciano, se recolectaron datos mediante el análisis de historias clínicas y prescripciones médicas y posteriormente se clasificaron según la WHOCC. Finalmente, los datos fueron procesados y tabulados utilizando el software Microsoft Office Exceß 2016 y analizados con la ayuda del software estadístico R-project. Resultados: Se evidenció que todos los ancianos usaban al menos un medicamento, con una prevalencia de ancianos polimedicados $(88,9 \%)$ y fármacos que actúan sobre el sistema nervioso $(29,6 \%)$, siendo la dipirona el fármaco con mayor frecuencia de prescripción (59,3\%). Conclusión: Estos resultados refuerzan la importancia de constituir un equipo interdisciplinario enfocado en la calidad de la atención brindada y, en consecuencia, despertar el interés en futuras investigaciones sobre el objeto de estudio, dado el aumento de la esperanza de vida de la población.

Palabras clave: Anciano, Hogares para ancianos, Prescripciones de medicamentos.

\section{INTRODUÇÃO}

O envelhecimento populacional é um fenômeno mundial, em razão das mudanças nas taxas de mortalidade e fecundidade capaz de provocar significativas alterações na estrutura etária da população (UNITED NATIONS, 2019).

A definição de ser idoso está vinculada a distintos critérios, o mais usual deles é estritamente cronológico. Para a Organização Mundial da Saúde (OMS), idoso é considerado a pessoa com 60 anos ou mais em países em desenvolvimento, como o Brasil, e com 65 anos ou mais em países desenvolvidos (OMS, 2015).

Essa mudança na configuração populacional, conhecida como transição demográfica, vem ocorrendo de forma acelerada nos países em desenvolvimento e, especialmente, no Brasil nas últimas cinco décadas, caracterizadas por um aumento da expectativa de vida, redução da mortalidade infantil, melhora nas condições de saúde, combate às doenças infectocontagiosas e queda na taxa de fecundidade. Estima-se que em 2050, a população brasileira idosa poderá chegar a 64 milhões de pessoas e deverá representar quase $30 \%$ da população ao final deste período (INSTITUTO BRASILEIRO DE GEOGRAFIA E ESTATÍSTICA (IBGE), 2019).

É importante reconhecer que a idade cronológica não é um marcador preciso para as mudanças que acompanham o envelhecimento. Existem diferenças significativas relacionadas ao estado de saúde, participação e níveis de independência entre pessoas que possuem a mesma idade (WORLD HEALTH ORGANIZATION, 2005).

As mudanças que constituem e influenciam o envelhecimento são multidimensionais. $O$ processo de envelhecimento leva a diminuição gradual da capacidade funcional de múltiplos órgãos e sistemas, aumento do risco de contrair diversas comorbidades clínicas e um declínio geral na capacidade intrínseca do indivíduo, influenciando nos aspectos relacionados à resposta do organismo aos medicamentos, ou seja, interferindo na farmacocinética e farmacodinâmica. A primeira delas se refere ao processamento do fármaco pelo corpo depois da administração, envolvendo a absorção, distribuição, metabolismo e excreção; já a segunda discute a ação do fármaco no corpo (BRENES-SALAZAR JA, et al., 2015). Essas alterações, associadas à polifarmacoterapia entendida como o uso concomitante de cinco ou mais medicamentos, tornam esse contingente populacional mais vulnerável a interações, aumento do risco de reações adversas e ineficácia terapêutica e/ou toxicidade quando comparados às demais camadas da população (ARAÚJO RQ, et al., 2013; OMS, 2019; SANTOS NS, et al., 2019).

Além disso, a idade avançada frequentemente envolve mudanças significativas além das perdas biológicas. Essas mudanças incluem alterações nos papéis e posições sociais, bem como nas modificações das estruturas familiares, ausência da família, ruptura de vínculos ou mesmo escassez de recursos financeiros para arcar com as despesas de contratação de um profissional de saúde para cuidar do idoso, podem levar a busca pela institucionalização, na chamada Instituição de Longa Permanência para Idoso (ILPI). Esses estabelecimentos podem ser mantidos pelo governo, por associações religiosas e beneficentes ou pelos próprios idosos e familiares, sendo muitas vezes alternativas de moradia aos que antes residiam sozinhos e 
àqueles cujos familiares não dispõem de estrutura para exercer o cuidado (BRASIL, 2003; OLIVEIRA MPF e NOVAES MRCG, 2013).

Nesse sentido, são consideradas fundamentais investigações que toquem a condição de saúde de idosos, especialmente os residentes em ILPI. Isso porque parte desta população convive com a falta de conhecimento acerca da sua condição de saúde que se constitui da interação de diferentes aspectos cronológicos, biológicos, psicológicos, sociais, históricos, políticos, econômicos, religiosos e culturais que determinam diversos efeitos nessa fase do ciclo da vida. Considerando esse pressuposto, esse estudo teve como objetivo avaliar a prevalência e fatores associados à prescrição de medicamentos em idosos institucionalizados.

\section{MÉTODOS}

Trata-se de um estudo de corte transversal, com delineamento descritivo e abordagem quantitativa com idosos residentes na única ILPI pública localizada na capital e de referência no estado de Roraima. Este estabelecimento de saúde apresenta capacidade para atender trinta idosos e tem como missão institucional prestar assistência aos idosos com variados graus de dependência funcional na execução de atividades de vida diárias e de vulnerabilidade social. Os idosos residentes na ILPI foram selecionados, de acordo com os seguintes critérios de inclusão: idade igual ou superior a 60 anos, ambos os sexos, que residiam na instituição há mais de trinta dias no período da coleta de dados. Foram excluídos do estudo os idosos que durante o período de coleta de dados estavam hospitalizados.

Os dados foram obtidos por meio de consulta direta aos prontuários e prescrições médicas de todos os idosos residentes, realizada semanalmente no período de dezembro de 2018. Foram considerados os seguintes elementos na produção dos achados: dados sociodemográficos (idade, sexo, tempo de institucionalização); condições clínicas do idoso (diagnósticos médicos) e dados farmacoterapêuticos (nome dos fármacos prescritos, posologia, via de administração e forma farmacêutica). Cabe destacar, que todos os dados foram solicitados junto ao responsável técnico da respectiva ILPI.

A análise farmacoterapêutica exigiu que os medicamentos que estavam prescritos com nomes comerciais fossem identificados por seus princípios ativos com o auxílio do Bulário Eletrônico da Agência Nacional de Vigilância Sanitária (ANVISA, 2020). Posteriormente, os princípios ativos de administração oral e parenteral encontrados em cada medicamento foram classificados conforme a Anatomical Therapeutic Chemical (ATC) da World Health Organization Collaborating Centre (WHOCC, 2019).

Nesta classificação, os princípios ativos são agrupados em cinco níveis diferentes, sendo o grupo anatômico principal e quatro subgrupos: terapêutico, farmacológico, químico e substância química. Neste estudo, os medicamentos foram agrupados e classificados conforme o primeiro, segundo e quinto nível de classificação da ATC (WHOCC, 2019).

É importante ressaltar que para a classificação ATC, foram desconsiderados fármacos de uso tópico, otológico, oftalmológico, fitoterápico e também suplementos alimentares. As doenças identificadas foram agrupadas de acordo com a Classificação Internacional de Doenças (CID-10) (BRASIL, 2019).

Por fim os dados foram processados e tabulados através do software Microsoft Office Exce ${ }^{\circledR} 2016$ e analisados com o auxílio do software estatístico R-projec ${ }^{\circledR}$. As análises estatísticas constituíram-se de: análises descritivas por meio de distribuição de frequências absolutas e percentuais para caracterizar o grupo em estudo e a utilização do teste de associação Qui-quadrado de Pearson para verificar associação estatisticamente significativa entre as variáveis do estudo, sendo o nível de significância adotado de $95 \%$ $(\mathrm{p}<0,05)$.

O estudo foi elaborado seguindo as normas e diretrizes definidas pelas Resoluções do Conselho de Saúde que regulamentam a realização de pesquisa envolvendo seres humanos no 466/2012 e no 510/2016 e foi aprovado pelo Comitê de Ética em Pesquisa da Universidade Federal de Roraima, sob o número do parecer 2.964.036. Além disso, foram respeitados os preceitos éticos e os pesquisadores assinaram o termo de proteção de risco e confidencialidade comprometendo-se a guardar sigilo das informações contidas nos prontuários e prescrições médicas e utilizá-los apenas para fins científicos. 
Tabela 2 - Classificação por grupo anatômico (1ํㅡível ATC $\left.{ }^{a}\right)$ e subgrupo terapêutico ( $2^{\circ}$ nível ATCa) de fármacos prescritos aos idosos institucionalizados $\mathrm{n}=81$.

\begin{tabular}{|c|c|c|c|}
\hline $\begin{array}{l}\text { ATC }^{\text {a }} 1^{\circ} \text { e } 2^{\circ} \\
\text { nível }\end{array}$ & Grupos e Subgrupos & $\mathbf{n}$ & $\%$ \\
\hline $\mathbf{N}$ & Sistema Nervoso & $24^{\mathrm{b}}$ & $29,6^{b}$ \\
\hline N06 & Psicoanalépticos & 8 & 9,9 \\
\hline N05 & Psicolépticos & 6 & 7,4 \\
\hline N02 & Analgésicos & 4 & 4,9 \\
\hline N03 & Antiepilépticos & 3 & 3,7 \\
\hline N07 & Outros medicamentos do sistema nervoso & 2 & 2,5 \\
\hline N4 & Medicamentos Anti-parkinsons & 1 & 1,2 \\
\hline C & Sistema Cardiovascular & $21^{\mathrm{b}}$ & $25,9^{b}$ \\
\hline $\mathrm{C03}$ & Diuréticos & 4 & 4,9 \\
\hline $\mathrm{C} 09$ & Agentes que agem sobre o sistema renina-angiotensina & 4 & 4,9 \\
\hline $\mathrm{C} 08$ & Bloqueadores de canais de cálcio & 3 & 3,7 \\
\hline C10 & Agentes modificadores de lipídeos & 3 & 3,7 \\
\hline $\mathrm{C} 07$ & Agente B-bloqueadores & 2 & 2,5 \\
\hline $\mathrm{C} 04$ & Vasodilatadores periféricos & 2 & 2,5 \\
\hline $\mathrm{C} 05$ & Vasoprotetores & 1 & 1,2 \\
\hline $\mathrm{C} 02$ & Anti-hipertensivos & 1 & 1,2 \\
\hline $\mathrm{C} 01$ & Terapia cardíaca & 1 & 1,2 \\
\hline A & Trato Alimentar e Metabolismo & $19^{b}$ & $23,5^{b}$ \\
\hline A10 & Medicamentos utilizados no diabetes & 7 & 8,6 \\
\hline A02 & Medicamentos relacionados a distúrbios com ácidos & 5 & 6,2 \\
\hline A03 & Medicamentos para transtornos gastrointestinais funcionais & 4 & 4,9 \\
\hline A06 & Medicamentos para constipação & 1 & 1,2 \\
\hline A09 & Digestivos incluindo enzimas & 1 & 1,2 \\
\hline A4 & Antieméticos e antinauseantes & 1 & 1,2 \\
\hline $\mathbf{R}$ & Sistema Respiratório & $7^{b}$ & $8,6^{b}$ \\
\hline R06 & Anti-histaminas para uso sistêmico & 3 & 3,7 \\
\hline R3 & Medicamentos para doenças das vias respiratórias obstrutivas & 3 & 3,7 \\
\hline R05 & Preparações de tosse e frio & 1 & 1,2 \\
\hline M & Sistema Músculo-Esquelético & $4 \mathrm{~b}$ & $4,9^{b}$ \\
\hline M01 & Produtos anti-inflamatórios e anti-reumáticos & 3 & 3,7 \\
\hline M05 & Medicamentos para o tratamento de doenças ósseas & 1 & 1,2 \\
\hline $\mathbf{J}$ & Anti-infecciosos para uso sistêmico & $3^{\mathrm{b}}$ & $3,7^{\mathrm{b}}$ \\
\hline J01 & Antibacterianos para uso sistêmico & 2 & 2,5 \\
\hline $\mathrm{J} 02$ & Antimicóticos para uso sistêmico & 1 & 1,2 \\
\hline $\mathbf{G}$ & Sistema Genito-Urinário e Hormônios Sexuais & $2^{b}$ & $2,5^{b}$ \\
\hline G04 & Urológicos & 2 & 2,5 \\
\hline H & $\begin{array}{c}\text { Preparações Hormonais Sistêmicas, Excluindo Hormônios } \\
\text { Sexuais e Insulinas }\end{array}$ & $1^{b}$ & $1,2^{b}$ \\
\hline \multirow[t]{2}{*}{$\mathrm{H} 02$} & Corticosteróides para uso sistêmico & 1 & 1,2 \\
\hline & Total & 81 & 100,0 \\
\hline
\end{tabular}

Legenda: a $\mathrm{ATC}=$ Anatomical Therapeutic Chemical; ${ }^{\mathrm{b}}$ Valores referentes às categorias dentro da classificação ATC. Fonte: Muniz TR, et al., 2021.

Baseado no $1^{\circ}$ e 2 nível da ATC, percebeu-se que os medicamentos do sistema nervoso $(29,6 \%)$ constituíram o grupo anatômico mais prevalente, sendo os psicoanalépticos $(9,9 \%)$ o subgrupo terapêutico mais utilizado, seguidos pelos medicamentos do sistema cardiovascular $(25,9 \%)$ e subgrupo terapêutico de fármacos com finalidade diurética e de agentes que agem sobre o sistema renina-angiotensina, ambos com frequência de $4,9 \%$.

Realizando ainda uma análise mais aprofundada, todos os medicamentos foram classificados de acordo

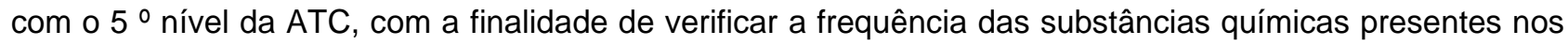
fármacos prescritos aos idosos. Verificou-se então que a dipirona sódica $(59,3 \%)$ apresentou maior frequência de prescrição entre os fármacos (Tabela 3). 
Tabela 3 - Distribuição das frequências de fármacos prescritos aos idosos institucionalizados, segundo o 5 nível da ATCa $n=81$.

\begin{tabular}{|c|c|c|c|}
\hline ATC $^{a} 5^{\circ}$ nível & Fármaco & $\mathbf{n}$ & $\%$ \\
\hline N02BB02 & Dipirona & 16 & 59,3 \\
\hline N06DX01 & Memantina & 10 & 37,0 \\
\hline N02BA01 & Ácido acetilsalicílico & 10 & 37,0 \\
\hline C09CA01 & Losartana & 9 & 33,3 \\
\hline M05BA04 & Alendronato de sódio & 9 & 33,3 \\
\hline C10AA01 & Sinvastatina & 8 & 29,6 \\
\hline N05AA02 & Levomepromazina & 8 & 29,6 \\
\hline N05AX08 & Risperidona & 8 & 29,6 \\
\hline A02BC01 & Omeprazol & 8 & 29,6 \\
\hline N06AB05 & Paroxetina & 7 & 25,9 \\
\hline C05CA53 & Diosmina+ Hisperidona & 7 & 25,9 \\
\hline C09CA06 & Candersatana & 7 & 25,9 \\
\hline C03CA01 & Furosemida & 6 & 22,2 \\
\hline M01AE01 & Ibuprofeno & 5 & 18,5 \\
\hline C07AG02 & Carvedilol & 5 & 18,5 \\
\hline C09AA01 & Captopril & 4 & 14,8 \\
\hline N06AA09 & Amitriptilina & 4 & 14,8 \\
\hline N03AA02 & Fenobarbital & 4 & 14,8 \\
\hline N05AD01 & Haloperidol & 4 & 14,8 \\
\hline A10BA02 & Metformina & 4 & 14,8 \\
\hline A03FA03 & Domperidona & 4 & 14,8 \\
\hline J01CR02 & Amoxicilina + clavulanato de potássio & 3 & 11,1 \\
\hline G04BD04 & Oxibutinina & 3 & 11,1 \\
\hline A03FA01 & Metoclopramida & 3 & 11,1 \\
\hline A03AX13 & Simeticona & 3 & 11,1 \\
\hline C04AE54 & Hidroergocristina + flunarizina & 3 & 11,1 \\
\hline A10BG03 & Pioglitazona & 3 & 11,1 \\
\hline N07CA03 & Flunarizina & 3 & 11,1 \\
\hline C08CA01 & Anlodipino & 2 & 7,4 \\
\hline
\end{tabular}




\begin{tabular}{|c|c|c|c|}
\hline ATC $^{a} 5^{\circ}$ nível & Fármaco & $\mathbf{n}$ & $\%$ \\
\hline C03DA01 & Espironolactona & 2 & 7,4 \\
\hline C03AA03 & Hidroclorotiazida & 2 & 7,4 \\
\hline C01AA05 & Digoxina & 2 & 7,4 \\
\hline N04BA01 & Levodopa & 2 & 7,4 \\
\hline N06AB04 & Citalopram & 2 & 7,4 \\
\hline N02AX02 & Tramadol & 2 & 7,4 \\
\hline N03AF01 & Carbamazepina & 2 & 7,4 \\
\hline R06AD02 & Prometazina & 2 & 7,4 \\
\hline J02AC01 & fluconazol & 2 & 7,4 \\
\hline $\mathrm{A} 10 \mathrm{BH} 01$ & Sitagliptina & 2 & 7,4 \\
\hline A10BB12 & Glimepirida & 2 & 7,4 \\
\hline A02BA02 & Ranitidina & 2 & 7,4 \\
\hline A02BC02 & Pantoprazol & 2 & 7,4 \\
\hline A06AA01 & Óleo mineral & 2 & 7,4 \\
\hline A10AB01 & Insulina regular & 1 & 3,7 \\
\hline A10AE04 & Insulina glargina & 1 & 3,7 \\
\hline A10AC01 & Insulina Humana Recombinante & 1 & 3,7 \\
\hline A02BC05 & Esomeprazol & 1 & 3,7 \\
\hline A02AD01 & Hidróxido de alumínio+magnésio & 1 & 3,7 \\
\hline A04AA01 & Ondansetrona & 1 & 3,7 \\
\hline A03DB04 & Escopolamina + dipirona & 1 & 3,7 \\
\hline A09AA04 & Lactase & 1 & 3,7 \\
\hline C10AB08 & Ciprofibrato & 1 & 3,7 \\
\hline C03BA11 & Indapamida & 1 & 3,7 \\
\hline C04AE01 & Codergocrina & 1 & 3,7 \\
\hline C09AA02 & Enalapril & 1 & 3,7 \\
\hline C10AA07 & Rosuvastatina & 1 & 3,7 \\
\hline C07AB03 & Atenolol & 1 & 3,7 \\
\hline C08CA05 & Nifedipino & 1 & 3,7 \\
\hline C08CA06 & Nimodipino & 1 & 3,7 \\
\hline
\end{tabular}




\begin{tabular}{|c|c|c|c|}
\hline ATC $^{a} 5^{\circ}$ nível & Fármaco & $\mathbf{n}$ & $\%$ \\
\hline N03AE01 & Clonazepam & 1 & 3,7 \\
\hline N07CA01 & Betaistina & 1 & 3,7 \\
\hline $\mathrm{N} 05 \mathrm{AH} 04$ & Quetiapina & 1 & 3,7 \\
\hline N05CF02 & Zolpidem & 1 & 3,7 \\
\hline N05BA06 & Lorazepam & 1 & 3,7 \\
\hline N02BE01 & Paracetamol & 1 & 3,7 \\
\hline N06AX21 & Duloxetina & 1 & 3,7 \\
\hline N06AA02 & Imipramina & 1 & 3,7 \\
\hline N06DA04 & Galantamina & 1 & 3,7 \\
\hline N06BX03 & Piracetam & 1 & 3,7 \\
\hline M01AX25 & Condroitina & 1 & 3,7 \\
\hline $\mathrm{M} 01 \mathrm{AH} 01$ & Celecoxibe & 1 & 3,7 \\
\hline G04CB01 & Finasterida & 1 & 3,7 \\
\hline R05CB06 & Ambroxol & 1 & 3,7 \\
\hline R03DC03 & Montelucaste de sódio & 1 & 3,7 \\
\hline R03DA08 & Bamifilina & 1 & 3,7 \\
\hline R03BA02 & Budesonida & 1 & 3,7 \\
\hline R06AX22 & Ebastina & 1 & 3,7 \\
\hline R06AE05 & Meclozina & 1 & 3,7 \\
\hline J01FF01 & Clindamicina & 1 & 3,7 \\
\hline C02CA04 & Doxazosina & 1 & 3,7 \\
\hline H02AB01 & Betametasona & 1 & 3,7 \\
\hline
\end{tabular}

Legenda: a $\mathrm{ATC}=$ Anatomical Therapeutic Chemical.

Fonte: Muniz TR, et al., 2021. 
Por fim, verificou-se mediante aplicação do teste de associação que nenhuma das variáveis apresentaram associação/influência estatisticamente significativa com a quantidade de medicamentos prescritos aos idosos institucionalizados (Tabela 4).

Tabela 4 - Associação entre medicamentos prescritos, características sociodemográficas e clínicas dos idosos institucionalizados $n=27$.

\begin{tabular}{|c|c|c|c|c|c|c|c|}
\hline \multirow{3}{*}{ Variável } & \multicolumn{6}{|c|}{ Medicamentos prescritos } & \multirow{3}{*}{ P-valor } \\
\hline & \multicolumn{2}{|c|}{$1-2$} & \multicolumn{2}{|c|}{$3-4$} & \multicolumn{2}{|c|}{$\geq 5$} & \\
\hline & $\mathbf{n}$ & $\%$ & $\mathbf{n}$ & $\%$ & $\mathbf{n}$ & $\%$ & \\
\hline \multicolumn{8}{|c|}{ Faixa etária } \\
\hline $60-69$ anos & 0 & 0 & 1 & 3,7 & 4 & 14,8 & \multirow{4}{*}{$0,3238^{a}$} \\
\hline $70-79$ anos & 0 & 0 & 1 & 3,7 & 11 & 40,7 & \\
\hline $80-89$ anos & 0 & 0 & 0 & 0 & 6 & 22,2 & \\
\hline 90 ou mais & 1 & 3,7 & 0 & 0 & 3 & 11,1 & \\
\hline \multicolumn{8}{|c|}{ Gênero } \\
\hline Feminino & 0 & 0 & 0 & 0 & 3 & 11,1 & \multirow{2}{*}{$1,0000^{a}$} \\
\hline Masculino & 1 & 3,7 & 2 & 7,4 & 21 & 77,7 & \\
\hline \multicolumn{8}{|c|}{ № de doenças diagnosticadas } \\
\hline 1 - 2 doenças & 1 & 3,7 & 1 & 3,7 & 15 & 55,5 & \multirow{3}{*}{$1,0000^{\mathrm{a}}$} \\
\hline 3 - 4 doenças & 0 & 0 & 1 & 3,7 & 8 & 29,6 & \\
\hline 5 ou mais & 0 & 0 & 0 & 0 & 1 & 3,7 & \\
\hline \multicolumn{8}{|c|}{ Tempo de institucionalização } \\
\hline $0-9$ anos & 0 & 0 & 2 & 7,4 & 18 & 66,7 & \multirow{3}{*}{$0,1305^{a}$} \\
\hline $10-19$ anos & 0 & 0 & 0 & 0 & 5 & 18,5 & \\
\hline 20 anos ou mais & 1 & 3,7 & 0 & 0 & 1 & 3,7 & \\
\hline Total & 1 & 3,7 & 2 & 7,4 & 24 & 88,9 & - \\
\hline
\end{tabular}

Legenda: P- significância estatística; a Teste Qui-quadrado de Pearson.

Fonte: Muniz TR, et al., 2021.

\section{DISCUSSÃO}

Os achados mostraram predomínio de idosos do sexo masculino $(88,9 \%)$ na ILPI. Essa prevalência difere de outras pesquisas nacionais, que demonstram um predomínio do sexo feminino nesse tipo de estabelecimento, justificada pela expectativa de vida maior das mulheres em relação aos homens (ARAÚJO NETO AH, et al., 2017 e ROSA TSM, et al., 2016).

A divergência existente entre os idosos homens com relação às mulheres é justificada pelo fato da ILPI abrigar, na maioria dos casos, homens, que mudaram para a região em busca de melhores condições de vida com a atividade no garimpo. Cabe sublinhar que a migração no estado de Roraima teve seu auge na década de 1980 com o garimpo, pois o mesmo era visto como uma possibilidade de enriquecimento através da extração mineral, ou seja, neste período houve um fluxo migratório de trabalhadores garimpeiros vindo de vários estados do país, que se direcionaram para Roraima e estabeleceram residência na capital, tanto na casa de parentes e amigos como em residências construídas com material disponibilizado pelo poder público que servia como base de apoio, sendo uma ocupação episódica de homens sozinhos e não de famílias, fundamentando deste modo, o provável motivo para a presença da maioria de idosos do sexo masculino na instituição (STAEVIE PM, 2011).

O perfil mais envelhecido neste estudo vai ao encontro do aumento da longevidade dos idosos brasileiros estimada pelo IBGE (2019). No entanto, a idade avançada traz consigo um aumento significativo da incidência de doenças crônicas e múltiplas aos idosos e que juntamente com as manifestações clínicas decorrentes do processo de envelhecimento influenciam no aumento a exposição à prática de polifarmácia (NECHBA RB, et al., 2015 e SKINNER M, 2015). 
Esses fatores levam a impactos clínicos negativos, como o aumento do risco de reações adversas, do potencial de interações medicamentosas, do número de medicamentos propriamente inapropriados, admissão hospitalar e também risco elevado de quedas (MARQUES GFM, et al., 2018).

Outro fator que pode contribuir para elevar o número de medicamentos utilizados por idosos é a prescrição distorcida ou impulsiva que ocorre em alguns casos, na tentativa de amenizar sintomas ou obter respostas mais rápidas em relação às medidas não farmacológicas (SILVEIRA EA, et al., 2014). Nesse contexto, podese dar início à cascata da prescrição, que ocorre quando os efeitos adversos dos medicamentos podem ser atribuídos de forma equivocada a uma condição médica e, dessa forma, um novo medicamento é prescrito para tratar um efeito adverso de outro medicamento, o que coloca o idoso institucionalizado em risco de desenvolver efeitos indesejáveis adicionais desnecessários com novas medicações, fatores esses que podem justificar a taxa elevada de polifarmácia $(88,9 \%)$ encontrada neste estudo e que difere da literatura (AMERICAN GERIATRICS SOCIETY, 2019 e AUGUSTO DK, 2018).

Estudos similares em ILPI revelam que $69,7 \%$ de idosos são polimedicados. No entanto, o resultado disposto no estudo não pode ser atribuído à prevalência de múltiplas doenças, visto que apenas $15,8 \%$ apresentavam mais de cinco diagnósticos o que também corrobora com os dados encontrados em Roraima. Apesar de apresentar uma elevada prescrição de medicamentos, a média encontrada de doenças diagnosticadas foi de 2,5 $\pm 1,4$ com destaque para as doenças cardiovasculares, dentre elas a hipertensão arterial sistêmica. Além disso, a baixa frequência de diagnósticos encontrados no presente estudo, podem ser justificados pelas informações desatualizadas em seus prontuários principalmente para aqueles idosos que residiam há mais tempo na instituição (REIS KMC e JESUS CAC, 2017).

A prevalência da HAS assemelha-se a de outras evidências científicas realizadas também com idosos residentes em ILPI (OLIVEIRA PC e NOVAES MRCG, 2013; RIBEIRO NP, et al., 2013). Essa doença constitui a morbidade mais frequente nos maiores de sessenta anos, sendo que cerca de dois terços dos idosos brasileiros são portadores de HAS, sua prevalência aumenta com a idade e os tratamentos inadequados favorecem a mortalidade por Acidente Vascular Encefálico (AVE) e Infarto Agudo do Miocárdio (IAM) (SECRETARIA DE ESTADO DE SAÚDE DE SÃO PAULO, 2011).

A sétima Diretriz Brasileira de Hipertensão Arterial da Sociedade Brasileira de Cardiologia (SBC) (2016), descreve que existe uma associação direta entre o envelhecimento e a prevalência de HAS e essa condição clínica pode ser agravada por outros fatores de risco dentre eles a presença de diabetes, que apresentou uma taxa de prevalência considerável no estudo.

As análises desta pesquisa apontam para uma concordância em relação à sequência do grupamento anatômico de ação dos fármacos com outros estudos. No estudo de Fochat RC, et al. (2011) o sistema nervoso liderou a categoria de medicamentos mais prescritos $(38,8 \%)$ e segundo Peixoto JS, et al. (2012) os medicamentos do sistema nervoso estão no topo dos mais prescritos $(37,8 \%)$, na sequência os que atuam no sistema cardiovascular (28,0\%), totalizando $65,8 \%$ das prescrições.

Com base nessas dimensões comparativas os sistemas mais prevalentes desta pesquisa também foram o nervoso e o cardiovascular. Sabe-se que o sistema nervoso é o sistema biológico mais comprometido com o processo do envelhecimento, por ser responsável pelas relações durante a vida, tais como sensações, movimentos, funções psíquicas, e ainda responde pela vida vegetativa, a qual envolve as funções biológicas internas do organismo (JESUS IS, et al., 2010).

Além disso, a prevalência do uso crônico de psicofármacos se dá devido à ansiedade e insônia que acometem os idosos institucionalizados; sintomas esses que ocorrem principalmente na utilização inadequada de medicamentos prescritos, o que também pode justificar o número expressivo de medicamentos prescritos que agem no sistema nervoso em relação aos cardiovasculares (OLIVEIRA MPF e NOVAES MRCG, 2013).

A dipirona liderou a classificação de fármacos mais prescritos aos idosos no período avaliado. Em seguida encontrou-se a memantina e ácido acetilsalicílico. A elevada prevalência de inflamação e dor entre os idosos, decorrentes principalmente das doenças crônicas, pode justificar a alta taxa de consumo de analgésicos 
nessa fase da vida (MONTEIRO SCM, et al., 2014). Outros achados científicos com este grupamento de idosos sinalizam que os principais agentes utilizados são: diazepam, fenotiazina, prometaziana e a fluoxetina (OLIVEIRA MPF e NOVAES MRCG, 2013; FOCHAT RC, et al., 2011).

No que diz respeito aos principais fármacos prescritos com ação no sistema cardiovascular foram evidenciados os antagonistas da angiotensina II (losartana) e as estatinas (sinvastatina). Em um estudo realizado com idosos aposentados no Brasil, estiveram entre os subgrupos predominantes, os diuréticos, os agentes com ação no sistema renina-angiotensina e os medicamentos para terapia cardíaca (SILVA JAC, et al., 2013).

O perfil medicamentoso encontrado neste estudo e na literatura de base reflete a realidade da população idosa, mais sujeita a ter doenças crônico-degenerativas como a hipertensão arterial e a insuficiência cardíaca, o que favorece a polifarmácia (LIRA KM, et al., 2015 e SILVA ACH, et al., 2014).

Cabe discutir que o omeprazol apresentou maior prevalência de prescrição entre os fármacos que atuam no sistema alimentar e metabólico. Este medicamento é muito empregado na atualidade cuja indicação é promover a cicatrização de úlceras gástricas ou duodenais e tratar a doença do refluxo gastroesofágico (MACIEL JC, 2015).

Aqui, observou-se porém, entre os idosos, apenas um diagnóstico compatível com a indicação para prescrição desse fármaco. Essa contradição segundo Silveira EA, et al. (2014) pode estar relacionada à ausência ou omissão de registros de comorbidades nos prontuários dos idosos ou como consequência também da polifarmácia, ou seja, a fim de aliviar sintomas gástricos associados ao uso excessivo de medicamentos e que também segundo a American Geriatrics Society (2015) seu consumo não deve exceder 8 semanas.

É preciso levar em consideração, que as diferenças na prevalência de medicamentos analisadas neste estudo, quando comparadas com os resultados de outras pesquisas, são atravessadas por peculiaridades nas condições de saúde, acesso aos serviços de atenção à saúde, além das próprias características demográficas e culturais ligados ao uso destes produtos entre as populações idosas (FOCHAT RC, et al., 2011). Tal fato demonstra a necessidade de adequações a um uso racional de medicamentos aos idosos institucionalizados.

As análises permitem ainda afirmar, que os achados divergem de estudos realizados com idosos não institucionalizados que notam associações da ocorrência de polifarmácia com variáveis demográficas como idade mais avançada e sexo feminino e também com variáveis clínicas, como a presença de comorbidades (CARNEIRO JA, et al., 2018; OLIVEIRA PC, et al., 2019; SALES AS, et al., 2017).

Logo é imprescindível a realização de estudos que identificam fatores associados ao consumo excessivo de medicamentos entre os idosos institucionalizados, considerando uma casuística segura. Acredita-se que esta relação possa contribuir na priorização de ações promocionais da saúde e prevenção dos agravos em idosos institucionalizados, visto a utilização excessiva de medicamentos na ILPI em Boa Vista-RR ser um fenômeno concretamente analisado.

Por fim é importante destacar que embora o presente estudo seja relevante em termos de caracterizar o perfil farmacoterapêutico de idosos institucionalizados na única instituição da capital de Roraima no extremo norte do país, o $\mathrm{N}$ amostral da pesquisa apesar de corresponder $96,4 \%$ da população estudada, possa não ser significativo e desta forma tenha influenciado no teste de associação. Pode-se destacar também, 0 delineamento transversal do estudo, que impossibilita o acompanhamento farmacoterapêutico dos idosos institucionalizados, uma vez que a coleta de dados ocorreu em um único momento no tempo.

\section{CONCLUSÃO}

As análises do perfil farmacoterapêutico na ILPI de Roraima avaliada permitiu concluir que todos os idosos faziam o uso de pelo menos um medicamento com prevalência em idosos polimedicados e fármacos que atuam no sistema nervoso, sendo a dipirona o fármaco com maior frequência de prescrição entre os idosos. 
Com a certeza do inacabado, é reconhecido que este assunto não se esgota neste estudo. Nesse prisma, espera-se que as análises postas sobre prevalência e fatores associados à prescrição de medicamentos em idosos institucionalizados possam subsidiar o planejamento dos cuidados de saúde com vistas atender as reais necessidades desta população, reduzir riscos à saúde e promover o bem-estar físico, mental e social desse grupo, haja vista o aumento de perspectiva de vida da população.

\section{REFERÊNCIAS}

1. AMERICAN GERIATRICS SOCIETY. American Geriatrics Society 2015 updated beers criteria for potentially inappropriate medication use in older adults. Journal of the American Geriatrics Society, 2015; 63 (11): $2227-46$.

2. AMERICAN GERIATRICS SOCIETY. American Geriatrics Society 2019 Beers Criteria® Update Expert Panel. American Geriatrics Society updated AGS Beers Criteria for potentially inappropriate medication use in older adults. Journal of the American Geriatrics Society, 2019; 67 (4): 674-694.

3. AGÊNCIA NACIONAL DE VIGILÂNCIA SANITÁRIA (ANVISA). Ministério da Saúde. Bulário Eletrônico [Internet]. 2020. Disponível em: https://consultas.anvisa.gov.br/\#/bulario/. Acessado em: 18 de novembro de 2020.

4. ARAÚJO NETO AH, et al. Quedas em idosos institucionalizados: riscos, consequências e antecedentes. Revista Brasileira de Enfermagem, 2017; 70 (4):752-8.

5. ARAÚJO RQ et al. Análise das interações fármacos $x$ nutrientes dentre os medicamentos mais prescritos em uma clínica geriátrica. Revista Brasileira de Nutrição Clínica, São Paulo, 2013; 28 (4): 306-10.

6. AUGUSTO DK. Curso cuidado a pessoa com multimorbidade e polimedicamentos. Belo Horizonte: Nescon/UFMG, 2018; 57p.

7. BRASIL. Lei $n^{0} 10.741$, de $1^{\circ}$ de outubro de 2003. Dispõe sobre o Estatuto do Idoso e dá outras providências. Brasília, DF: Secretaria Especial dos Direitos Humanos [Internet]. 2003 Disponível em: http://www.planalto.gov.br/ccivil_03/leis/2003/l10.741.htm. Acessado em: 8 de junho de 2020.

8. BRASIL. Ministério da Saúde. Departamento de Informática do SUS-DATASUS. Classificação Estatística Internacional de Doenças e Problemas Relacionados à Saúde [Internet]. Disponível em: http://www.datasus.gov.br. Acessado em: 02 de novembro de 2020.

9. BRENES-SALAZAR JÁ, et al. Clinical pharmacology relevant to older adults with cardiovascular disease. Journal of Geriatric Cardiology, 2015; 12(3): 192-195.

10. CARNEIRO JA, et al. Prevalência e fatores associados à polifarmácia em idosos comunitários: estudo epidemiológico de base populacional. Medicina, Ribeirão Preto, 2018; 5 (4):254-264.

11. FOCHAT RC, et al. Perfil de utilização de medicamentos por idosos frágeis institucionalizados na Zona da Mata Mineira, Brasil. Revista de ciências farmacêuticas básica e aplicada, 2012; 33(3): 447-54.

12. INSTITUTO BRASILEIRO DE GEOGRAFIA E ESTATÍSTICA (IBGE). Projeção da população do Brasil e das Unidade da Federação. 2019. Disponível em: https://www.ibge.gov.br/apps/populacao/projecao/index.html. Acessado em: 14 de novembro de 2020.

13. JESUS IS, et al. Cuidado sistematizado a idosos com afecção demencial residentes em instituição de longa permanência. Revista Gaúcha de Enfermagem, Porto Alegre, 2010; 31(2): 285-92.

14. LIRA KM, et al. Utilização de medicamentos potencialmente inapropriados para idosos atendidos em uma UBS do município de Cuíte/PB. Anais CIEH, 2015; 2 (1).

15. MACIEL JC. Uso racional de agentes supressores de ácido para refluxo gastroesofágico. In: Semana da Enfermagem da Universidade Federal de Roraima, Boa Vista, 2015; 1.

16. MARQUES GFM, et al. Polifarmácia e medicamentos potencialmente inapropriados para idosos na enfermagem gerontológica. Revista Brasileira de Enfermagem, 2018; 71 (5): 2440-6.

17. MONTEIRO SCM, et al. Automedicação em idosos de um Programa Saúde da Família, Brasil. Infarma, $2014 ; 26$ (2): 90-95.

18. NECHBA RB, et al. Difficulty in managing polypharmacy in elderly: case report and review of the literature. Journal of Clinical Gerontology \& Geriatrics, 2015; 6 (1): 30-33.

19. OLIVEIRA MPF, NOVAES MRCG. Perfil socioeconômico, epidemiológico e farmacoterapêutico de idosos institucionalizados de Brasília, Brasil. Ciência \& Saúde Coletiva, 2013; 18(4): 1069-1078.

20. OLIVEIRA PC, et al. Prevalência e Fatores Associados à Polifarmácia em Idosos Atendidos na Atenção Primária à Saúde Em Belo Horizonte, MG. Ciência \& Saúde Coletiva, 2019.

21. ORGANIZAÇÃO MUNDIAL DE SAÚDE (OMS). Relatório mundial de envelhecimento e saúde. 2015. Disponível em: https://sbgg.org.br/wp-content/uploads/2015/10/OMS-ENVELHECIMENTO-2015-port.pdf. Acessado em: 15 de junho de 2020.

22. PEIXOTO JS, et al. Riscos da interação droga-nutriente em idosos de instituição de longa permanência. Revista Gaúcha de Enfermagem, Porto Alegre, 2012; 33 (3): 156-164.

23. REIS KMC, JESUS CAC. Relação da polifarmácia e polipatologia com a queda de idosos institucionalizados. Texto \& Contexto Enfermagem, 2017; 26 (2): e3040015.

24. RIBEIRO NP, et al. Polifarmácia utilizada por idosos residentes em instituições de longa permanência do município de Viamão/RS. Ciência em Movimento, 2013; 15(30): 65-74.

25. ROSA TSM, et al. The institutionalized elderly: sociodemographic and clinical-functional profiles related to dizziness. Brazilian Journal of Otorhinolaryngology, 2016; 82 (2): 159-169. 
26. SALES AS, et al. Perfil farmacoterapêutico e fatores associados à polifarmácia entre idosos de Aiquara, Bahia, em 2014. Epidemiologia e Serviços de Saúde, 2017; 26 (1): 121-132.

27. SANTOS NS, et al. Intervenções para reduzir a prescrição de medicamentos inapropriados para idosos. Revista de Saúde Pública, 2019; 53:7.

28. SECRETÁRIA DE ESTADO DA SAÚDE DE SÃO PAULO. Gabinete do Secretário. Assessoria Técnica. Manual de orientação clínica: hipertensão arterial sistêmica (HAS). São Paulo: SES/SP [Internet], 2011, 68p. Disponível em: http://www.saude.sp.gov.br/resources/ses/perfil/gestor/destaques/linhas-de-cuidado-sessp/hipertensao-arterialsistemica/manual-de-orientacao-clinica-de-hipertensao-arterial/lc_hipertensao_manual_2011.pdf. Acessado em: 08 de dezembro de 2020.

29. SILVA ACH, et al. Medicamentos usados por idosos e critério de Beers e colaboradores. Diagnóstico e Tratamento, $2014 ; 19(3)$

30. SILVA JAC, et al. Prevalência de automedicação e os fatores associados entre os usuários de um Centro de Saúde Universitário. Revista da Sociedade Brasileira de Clínica Médica, 2013; 11 (1): 27-30.

31. SILVEIRA EA, et al. Polifarmácia, doenças crônicas e marcadores nutricionais em idosos. Revista Brasileira de Epidemiologia, 2014; 17 (4): 818-829.

32. SKINNER, M. A literature review: polypharmacy protocol for primary care. Geriatric Nursing, London, 2015; 6(5): 367371.

33. SOCIEDADE BRASILEIRA DE CARDIOLOGIA (SBC). VII Diretriz Brasileira de Hipertensão arterial. Arquivos Brasileiros de Cardiologia [Internet], 2016; 107(3). Disponível em: http://publicacoes.cardiol.br/2014/diretrizes/2016/05_HIPERTENSAO_ARTERIAL.pd. Acessado em: 8 de dezembro de 2020.

34. STAEVIE PM. Migrações e múltiplas identidades dos imigrantes em Boa Vista-RR. Informe Gepec, Toledo, $2011 ; 15$ (3): 478-487.

35. UNITED NATIONS. Department of Economic and Social Affairs, Department of Economic and Social Affairs. World Population Prospects 2019: Highlights. New York: USA, 2019.

36. WORLD HEALTH ORGANIZATION (WHO). Envelhecimento ativo: uma política de saúde / World Health Organization; tradução Suzana Gontijo. - Brasília: Organização Pan-Americana da Saúde, 2005. p. 60.

37. WORLD HEALTH ORGANIZATION COLLABORATING CENTRE FOR DRUG STATISTICS METHODOLOGY (WHOCC). ATT/DDD Index [Internet]. 2019. Disponível em: https://www.whocc.no/atc_ddd_index/. Acessado em: 13 de setembro de 2020. 